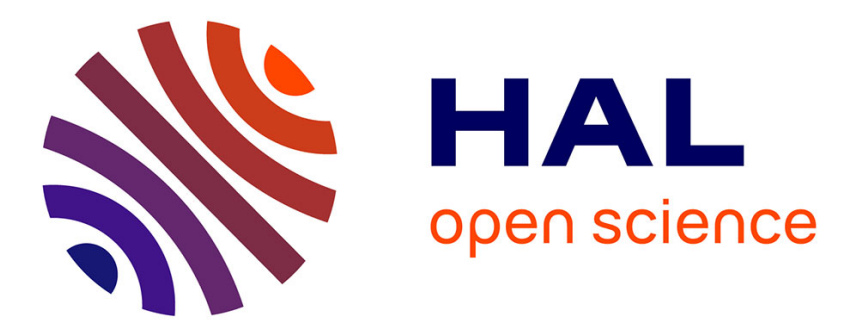

\title{
A process performance index based on gate-distance and incubation time for the optimization of gate locations in liquid composite molding processes
}

\author{
F. Sánchez, J.A. García, Francisco Chinesta, Ll. Gascón, C. Zhang, Z. Liang, \\ Biao Wang
}

\section{To cite this version:}

F. Sánchez, J.A. García, Francisco Chinesta, Ll. Gascón, C. Zhang, et al.. A process performance index based on gate-distance and incubation time for the optimization of gate locations in liquid composite molding processes. Composites Part A: Applied Science and Manufacturing, 2006, 37 (6), pp.903 - 912. 10.1016/j.compositesa.2005.01.016 . hal-01004932

\section{HAL Id: hal-01004932 \\ https://hal.science/hal-01004932}

Submitted on 19 Feb 2017

HAL is a multi-disciplinary open access archive for the deposit and dissemination of scientific research documents, whether they are published or not. The documents may come from teaching and research institutions in France or abroad, or from public or private research centers.
L'archive ouverte pluridisciplinaire HAL, est destinée au dépôt et à la diffusion de documents scientifiques de niveau recherche, publiés ou non, émanant des établissements d'enseignement et de recherche français ou étrangers, des laboratoires publics ou privés. 


\title{
A process performance index based on gate-distance and incubation time for the optimization of gate locations in liquid composite molding processes
}

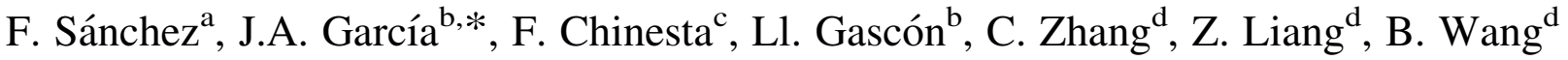 \\ ${ }^{a}$ Universidad Cardenal Herrera CEU, Escuela Superior de Enseñanzas Técnicas, C/San Bartolomé 55, E-46115 Alfara del Patriarca, Valencia, Spain \\ ${ }^{\mathrm{b}}$ Universidad Politécnica de Valencia, Ing. Mecanica y Materiales, Camino Vera s/n, 46022 Valencia, Spain \\ 'Laboratoire de Mécanique des Systèmes et des Procédés, UMR 8106 CNRS-ENSAM-ESEM, 151 Boulevard de l'Hôpital, F-75013 Paris, France \\ ${ }^{\mathrm{d}}$ Department of Industrial Engineering, FAMU-FSU College of Engineering, 2525 Pottsdamer Street, Tallahasse, FL 32310-6046, USA
}

\begin{abstract}
The success of filling and curing stages in a liquid composite molding (LCM) depends on many variables such as locations of gates and vents, temperature distribution, flow rate, injection pressure, etc. A great challenge to obtain high quality finished parts is to accurately predict flow pattern, and temperature and conversion profiles using simulation for process design optimization. With the predicted process performance measures, the LCM process can be optimized through locating the gates and vents properly.

In this study, a process performance index based on gate-distance and incubation time for liquid composite molding processes is defined. This index allows the consideration of both resin flow and curing issues in process design optimization. Another advantage in using this index in process optimization is that the computation involved is very efficient as only two-dimensional calculation is required. For this purpose, we make use of a new and accurate high order discretization technique for the filling flow kinematics-incubation time evolution coupled model, presented in some of our former works. This makes the index very useful in LCM process design optimization, where costly iterative stochastic search algorithms such as genetic algorithms are used. The effectiveness of the approach is illustrated through a number of examples which involve race tracking, areas with different permeabilities, different filling conditions, etc.
\end{abstract}

Keywords: C. Computational modelling; E. Resin transfer moulding (RTM); E. Resin flow; E. Cure

\section{Introduction}

Liquid Molding Processes and, particularly, Resin Transfer Molding (RTM), are being widely used in the manufacturing of fiber-reinforced composite materials. These processes are based on the preform reinforcement impregnation with a low viscosity resin, from which composites parts are conformed after the curing reaction (see Fig. 1). Most applications of the process require a short manufacturing cycle time and low production costs. An appropriate design of the process can be carried out with virtual manufacturing tools such as computer simulations.

Many of the final qualities of the piece are determined during the injection and curing stages. An accurate prediction of the mold flow pattern, pressure distribution, temperature and curing profile of the resin using simulation techniques allow one to optimize the process and, hence, to improve the final properties of the manufactured part. The design variables (process inputs) such as injection pressure, injection flow rate, gate and vent locations, mold and resin temperatures, etc. are introduced in the optimization models. Based on the manufacturing objectives, the optimization algorithms determine iteratively the optimal values of the process parameters. Nevertheless, due to the increasing complexity of manufactured parts and to the time required to simulate many of the cases like the threedimensional non isothermal filling, simulation times 


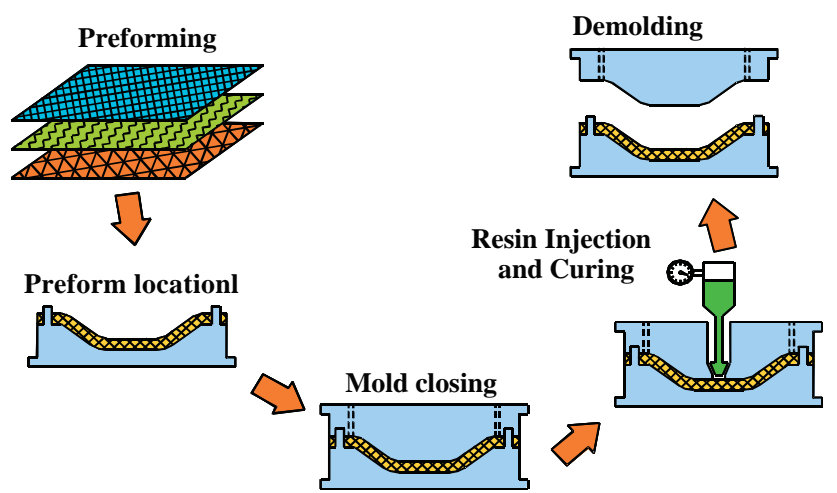

Fig. 1. Illustration of the stages in resin transfer molding processes.

involving optimisation become excessive. In this manner, the introduction of efficient and accurate simulation tools within the optimization algorithms will be a key issue in composites manufacturing.

\subsection{RTM mold filling simulation}

The resin flow through a porous medium can be modelled by Darcy's law

$\underline{\nu}=-\underline{\underline{\underline{K}}} \nabla p$

where $\underline{\nu}$ is the velocity, $\underline{\underline{K}}$ is the preform permeability tensor, $\mu$ is the fluid viscosity and $p$ is the pressure. The fluid flow problem is defined in a volume $\Omega$

$\Omega=\Omega_{\mathrm{f}}(t) \cup \Omega_{\mathrm{e}}(t)$

where the fluid at time $t$ occupies the volume $\Omega_{\mathrm{f}}(t)$ and $\Omega_{\mathrm{e}}(t)$ defines at that time the empty part of the mold. Assuming constant permeability and viscosity, orthotropic preform and the fluid incompressibility, the variational formulation related to the Darcy flow results

$$
\int_{\Omega_{\mathrm{f}}(t)}\left(\nabla p^{*} \cdot \underline{\underline{K}} \nabla p\right) \mathrm{d} \Omega=0
$$

where $p^{*}$ denotes the usual weighting function.

The prescribed conditions to impose on the boundary of $\Omega_{\mathrm{f}}(t)$ are:

- The pressure gradient in the normal direction to the mold walls is zero.

- The pressure or the flow rate is specified at the injection nozzle.

- Zero pressure is applied on the flow front.

The flow kinematics is computed by means of a conforming finite element Galerkin technique applied to the variational formulation extended to the whole domain $\Omega$ [1], imposing null pressure at the nodes not connected with at least a completely filled element.
An important concern in the mold filling simulation is the numerical treatment of the moving boundary defined by the flow front of the liquid resin. The domain occupied by the fluid where the governing equations have to be integrated changes continuously, so it has to be defined at each time step during the simulation. The fluid domain evolution is accomplished by the resolution of the hyperbolic transport equation that governs the fluid presence function $I$ updating

$\frac{\mathrm{d} I}{\mathrm{~d} t}=\frac{\partial I}{\partial t}+\underline{\nu} \cdot \nabla I=0$

with $I$ defined by:

$I(\underline{x}, t)= \begin{cases}1 & \underline{x} \in \Omega_{\mathrm{f}}(t) \\ 0 & \underline{x} \notin \Omega_{\mathrm{f}}(t)\end{cases}$

The numerical resolution of hyperbolic equations has been extensively treated by different authors due to the difficulty to obtain schemes that reproduce with accuracy the solution in both smooth regions and in the presence of discontinuities, see for instance [2,3]. First order upwind techniques have been used to approximate solutions of conservation laws. These methods have a strong numerical dissipation in the neighbourhood of discontinuities giving a low accuracy in smooth regions of the solution, which means in RTM a diffusive definition of the flow front. In order to avoid this problem, some second-order finite difference schemes have been proposed. The Total Variation Diminishing (TVD) schemes were introduced by Harten [4]. A particular version of these techniques is the flux limiters introduced by Sweby [5], based on the definition of hybrid schemes that use second-order approximations of the solution in the smooth regions and a limitation of the numerical fluxes to the first order in the vicinity of the discontinuities avoiding the spurious oscillations. Its implementation applied to RTM was issued in [1] and a deeper analysis was completed in [6].

\subsection{The incubation time as a curing-related simulation variable}

Another important issue in the numerical simulation is the resin curing stage. In LCM, the goal is to saturate completely the fiber preform with the resin before the curing reaction reaches the threshold value where the viscosity starts to increase abruptly, also known as gelling point. The chemical reaction begins when the pre-polymers are mixed just before the injection in the mold cavity (see Fig. 2). In many cases, the resins need to be heated to initiate the cure, nevertheless, the use of the inhibitors in the resin formulation allows to control and delay the curing [7,8] and hence, an isothermal mold filling is possible. Also, if a multi-component injection system is used, one could change the initial concentration of the resin catalyst in the mixing head with a controlled system (see Fig. 3). Then the catalyst concentration may be different 


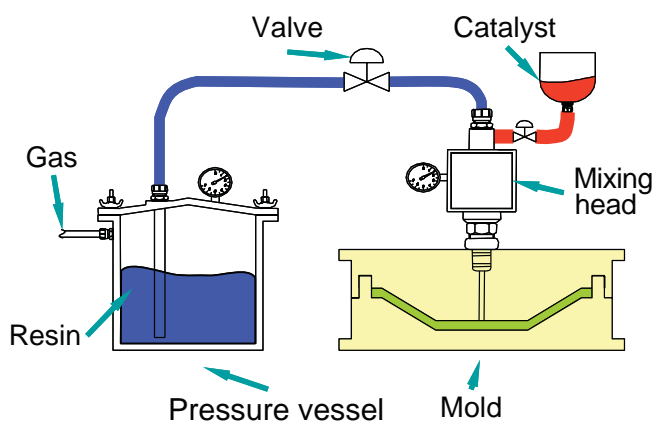

Fig. 2. Schematic resin injection system with constant resin formulation.

for each region of the filled mold yielding different rates of cure. In any case, it is important to prevent early gelation and avoid nonhomogeneous curing.

Mold filling constraint can be defined enforcing that the resin gelling time must be higher than the mold filling time, that is

$t_{\mathrm{gel}}-t_{\text {fill }}>0$

Moreover, the resin gelling time can be delayed and controlled by using different inhibitor concentrations in the polymerization resin agents as the fluid flows, see for instance $[9,10]$. In [10], Comas-Carmona et al. used the correlation of the gelling time and the concentration of the inhibitor as follows

$\ln \left(t_{\text {gel }}\right)=a+b C_{\text {hardener }}+c C_{\text {accelerator }}+d C_{\text {inhibitor }}$

where $a, b, c, d$, are constants to be determined for each resin, and $C$ refers to the concentration of the different chemical agents.

On the other hand, the curing reaction proceeds since the pre-polymers are mixed and its evolution depends mainly on the temperature and time. During the mold filling stage, the curing conversion must not reach the gelling point value $\alpha_{\text {gel }}$ allowing a complete filling. Once the filling is finished, the curing continues and the part can be demolded when it has reached enough strength. For engineering purposes such as process control and optimization, the cure-kinetic reaction can be modeled as a function of time through

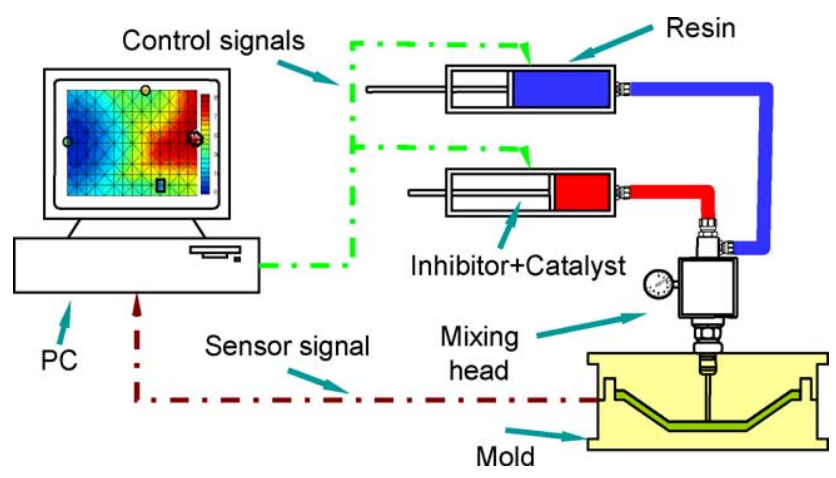

Fig. 3. Schematic resin injection system with PC-based control providing automatic mixing of resin components at injection gate. the temperature history [11]

$\alpha(t)=\sum_{i=1}^{N} a_{i} \exp \left(-\int_{0}^{t} k_{i}(t) \mathrm{d} t\right)$

where $a_{i}$ and $k_{i}$ depends on the resin system and $\alpha$ is the resin curing conversion factor.

The time to gel and the curing conversion depend directly on the resin formulation as described in Eqs. (7) and (8), but this topic is not addressed in the present work. In all the cases, the curing reaction depend on the time elapsed since the reactive mixture just before the injection, that is, on the incubation time. Moreover, if we consider an isothermal filling due to the use of inhibitors, then, the incubation time is directly related to the resin curing conversion as illustrated later. Therefore, the incubation time resolution can be used as a curing related variable for optimization and control purposes.

The main point of this approach is its generality (for all the chemical system), despite the fact that the incubation time is not the most appropriate way to describe the real curing evolution. Thus, by solving the incubation time variable one could detect improper processes. For example high gradients in the incubation time implies high gradient in the curing rates and consequently local thermomechanical stresses. In this work, we will solve the incubation time in different systems, without considering their chemical particularities.

The incubation time depends on the traveled path of each fluid particle throughout the mold since its injection, and therefore, is not always easily predicted. In many particular cases, such as multi-component injection systems, areas with different fiber permeabilities, complex geometries invloving obstacles, etc. the incubation time of the resin located in the flow front cannot be the injection time.

The calculation of the incubation time $E$ distribution throughout the mold has been achieved by solving the linear advection equation given by

$\frac{\mathrm{d} E}{\mathrm{~d} t}=\frac{\partial E}{\partial t}+\underline{\nu} \cdot \nabla E=1$

The value of the incubation time $E$ is zero in the injection nozzle (or a fictitious time accounting for the inhibitors presence, as described later, in controlled processes) and varies throughout the filled part of the mold, but it is not defined on the empty one

$E(\underline{x}, t)= \begin{cases}E(\underline{x}, t) & \underline{x} \in \Omega_{\mathrm{f}}(t) \\ \text { Not defined } & \underline{x} \notin \Omega_{\mathrm{f}}(t)\end{cases}$

The incubation time simulation depends directly on the previous resolution of the volume fraction, which allows locating the flow front position of the injected resin. The accurate resolution of both coupled advection problems has been implemented (see [12,13] for details) using a similar strategy for both equations based on the original definition 


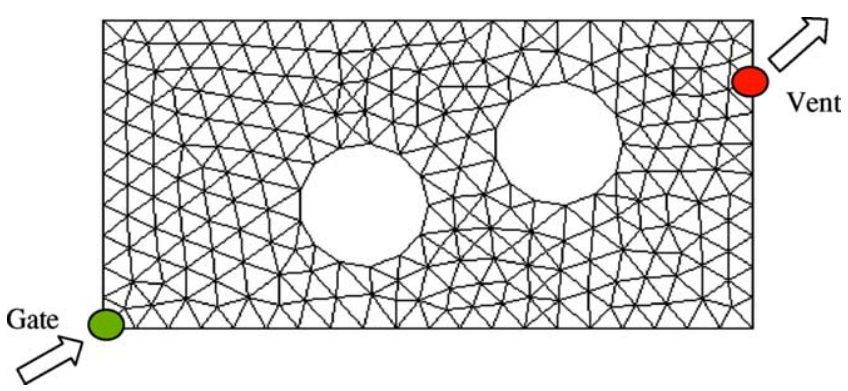

Fig. 4. Finite element mold discretization and location of the injection gate and vent.

and implementation of a second-order scheme with flux limiters applied to the integration of a general linear advection equation

$\frac{\mathrm{d} J}{\mathrm{~d} t}=\frac{\partial J}{\partial t}+\underline{\nu} \cdot \nabla J=S$

where $J=I$ and $S=0$ in the volume fraction updating case and we consider $J=E I$ and $S=I$ with the initial conditions

$(E I)(\underline{x}, t)= \begin{cases}E(\underline{x}, t) & \underline{x} \in \Omega_{\mathrm{f}}(t) \\ 0 & \underline{x} \notin \Omega_{\mathrm{f}}(t)\end{cases}$

for the integration of the incubation time. This last strategy proposed by Chinesta et al. [14] allows one to circumvent the particular difficulty of the incubation time not being defined in the empty part of the mold.

In order to illustrate the use of the incubation time as a resin curing design parameter, we can analyze some RTM simulations obtained in a recent work [6] for the mold depicted in Fig. 4. In this case, a mold of $1000 \mathrm{~mm} \times$ $500 \mathrm{~mm}$, with a thickness of $5 \mathrm{~mm}$, a permeability of $10^{-7} \mathrm{~m}^{2}$ containing $50 \%$ fiber volume, is considered. The resin viscosity of $1 \mathrm{~Pa} \mathrm{~s}$, and a constant flow injection rate of $5 \mathrm{~cm}^{3} / \mathrm{s}$, is considered. The circular obstacles induce velocity variations as well as the welding of different flow fronts. The injection gate is located in the lower left corner and the vent near the upper right one. Fig. 5 shows the flow pattern, which is the time at which the resin reaches each position. Fig. 6 represents the incubation time distribution. High values of the time can be noticed not only in the flow front position but also in the region with very small velocities like downstream the mold obstacles. Both results have been calculated with the simulation code FLOWSIM developed in our research

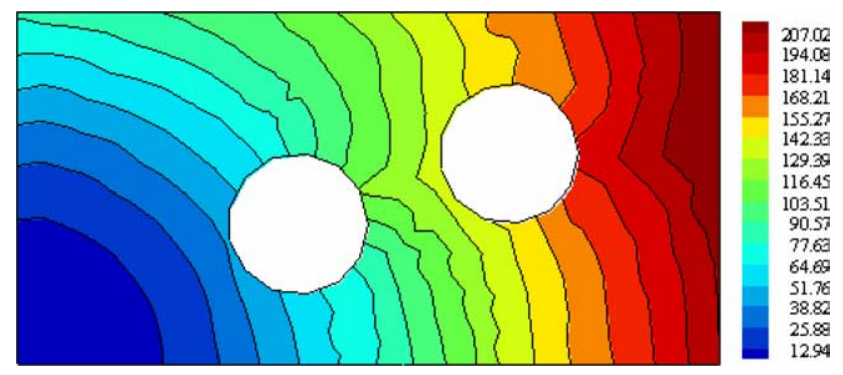

Fig. 5. Flow pattern.

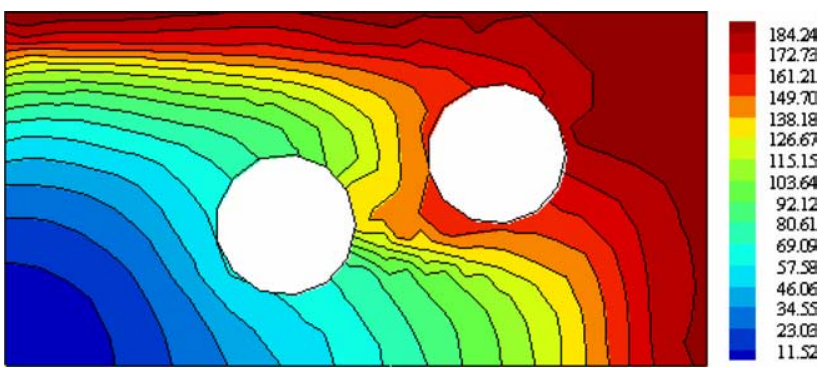

Fig. 6. Incubation time distribution at the end of the mold filling.

group in $[1,6,15]$. Fig. 7 depicts the curing conversion predicted with the commercial code CMOLD for the same process conditions. Assuming a curing conversion $\alpha_{\text {gel }}=0.2$ for the resin system, it is guaranteed the total mould filling before the viscosity buildup.

The knowledge of the resin flow front evolution and incubation time requires only a two-dimensional isothermal simulation. The main advantage in considering the isothermal case is not only the lower CPU time required, but also the fact that the number of material parameters involved in the model is reduced with respect to those required in non-isothermal three-dimensional simulations.

Several computer simulations, including some which address the thermal and curing problems, have been developed in RTM process in [16-20].

\subsection{Related works in RTM process optimization}

Many research studies have been focused on the use of simulations in the RTM process optimization. We can find different optimization models in which the maximum temperature differences have been considered $[19,21]$. The use of neural networks and genetic algorithms has been also applied with success in [22-26]. With these techniques, iterative stochastic search algorithms are required and then, the computation involved is very important. In other recent work [27], the optimization problem is treated by using the branch and bound search method. In all these cases, the process optimization objective functions, such as minimum filling time or dry spot prevention, have to be defined quantitatively.

In this work, a new process performance index is presented. The use of the incubation time as a curing-related

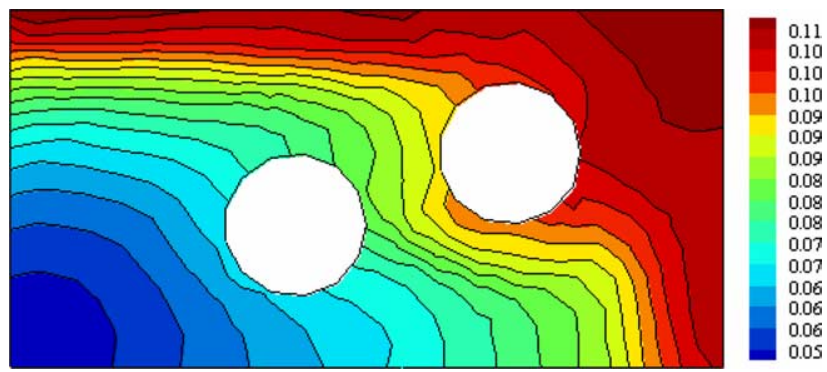

Fig. 7. Curing conversion at the end of the mold filling. 
variable allows optimizing the LCM process, locating the gates and vents properly in order to reduce cycle time, avoid dry spots and obtain a spatially homogenized curing. This effectiveness of the approach is illustrated through a number of examples which involve race tracking, areas with permeabilities, different filling conditions, etc.

\section{Definition of the resin flow index based on gate distance and incubation time}

The success of filling and curing stages in liquid composite molding (LCM) depends on many variables such as locations of gates and vents, temperature distribution, flow rate, injection pressure, etc. Traditionally the selection of gate and vent locations in mold design is based on experience and trial and error attempts. Recent research studies have been conducted to reduce cycle time by using computer simulation and optimization. Zhang et al. employed in [23-26] a process performance index based on gate-distance of the resin located on the flow front at different time steps. A good process should have short filling time and a vent-oriented flow with a desired resin flow pattern. At a given time step, the distances from the nodes located on the resin flow front to the outlet are associated with the quality of the filling process (Fig. 8). The standard deviation of those distances is used to evaluate the shape of the flow front (the smaller the better).

In addition to the shape of the flow pattern, total mold filling time is included to guarantee overall performance of a mold filling process. A process performance index was proposed as follows

$Q=\frac{T \times \sum_{k=1}^{m} \sqrt{\frac{\sum_{i_{k}=1}^{n_{k}}\left(d_{i k}-\bar{d}_{k}\right)^{2}}{n-1}}}{m}=\frac{T \times \sum_{k=1}^{m} q_{k}}{m}$

where

$Q$ overall process performance index (the lower the better)

$T$ total mold filling time

$q_{k}$ intermediate flow front index

$m$ number of flow fronts considered $n_{k}$ number of nodes defining the $k$ th flow front

$d_{i k}$ distance from node $i$ located on the $k$ th flow front to the outlet

$\bar{d}_{k}$ average distance from the nodes defining the $k$ th flow front to the outlet

The filling flow pattern is divided into $m$ time steps, and intermediate flow front indexes $q_{k}$ (standard deviation) can be calculated for every flow front considered which is associated to the time step $k$. Then the overall process performance index $Q$ is calculated from the intermediate values $q_{k}$ as illustrated in Eq. (13).

This index has been extended in this work in order to include the incubation time as a variable related with an optimal curing performance. The new index is defined as follows

$Q D=\frac{T \times D \times \sum_{k=1}^{m} q_{k}}{m}, \quad D=\frac{\sum_{i=1}^{n_{f}} D_{i}}{n_{\mathrm{f}}} ;$

$D_{i}=\max _{j=1}^{n n_{i}}\left(E_{j}\right)-\min _{j=1}^{n n_{i}}\left(E_{j}\right)$

where:

$Q D$ overall process performance index (the lower the better)

$T$ total mold filling time

$q_{k}$ intermediate flow front index

$m$ number of flow fronts considered

$D$ resin incubation time dispersion index defined for all filled nodes at the end of mold filling

$D_{i}$ dispersion index related to node $i$ which is assumed fullfilled

$E_{j} \quad$ incubation time for node $j$

$n n_{i}$ number of nodes connected with node $i$

$n_{\mathrm{f}} \quad$ number of filled nodes

Thus, the overall process performance index $Q D$ includes the consideration of the flow front shapes at different time steps with $q_{k}$, the total mold filling time $T$ and the differences in the incubation time values of all the nodes impregnated by the resin by means of the resin incubation time dispersion index $D$. For every filled node $i$, its dispersion index $D_{i}$ is calculated as the maximum difference

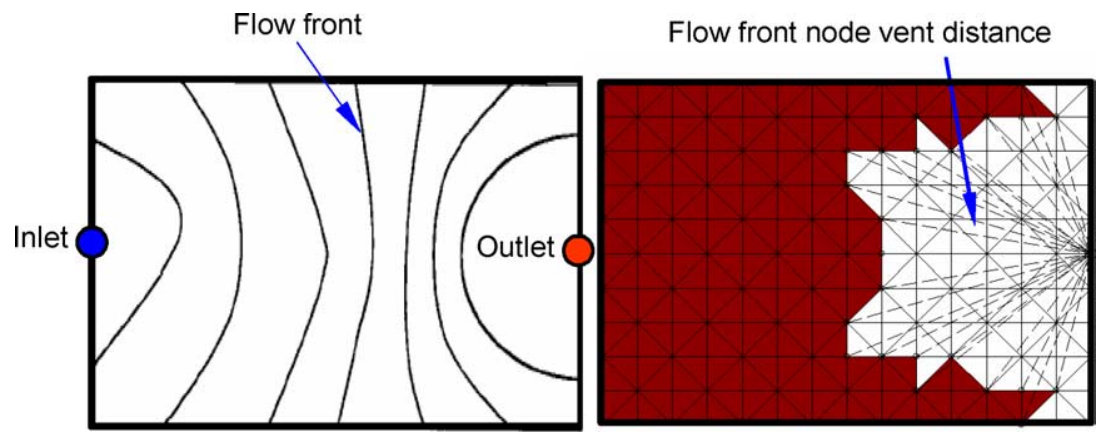

Fig. 8. Desired flow front shape (left) and distances between flow front nodes and vent (right). 


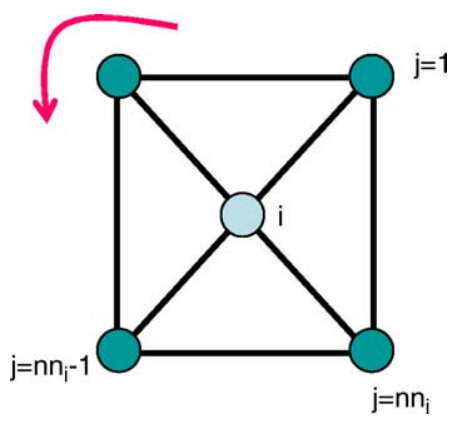

Fig. 9. Dispersión index $D_{i}$ definition.

of the incubation time values $E_{j}$ for all the nodes $j$ connected to node $i$, see Fig. 9. We note that the dispersion index $D_{i}$ accounts for the local differences in the incubation time, and therefore, in the curing conversion. A spatially homogeneous curing for the whole resin filled part of the mold is then conditioned by a small value of the resin incubation time dispersion index $D$. According to the previous definition, a good RTM process should have a desirable flow front shape, short filling time and small resin incubation time dispersion index.

\section{Numerical simulations}

In order to validate the resin flow index just presented, different numerical simulation experiments have been carried out to check how the index represents the RTM process behavior. In all cases, the injection process is defined in a mold of $140 \mathrm{~mm} \times 100 \mathrm{~mm}$, with a thickness of $6.4 \mathrm{~mm}$, a permeability of $2.714 \times 10^{-10} \mathrm{~m}^{2}$ with $50 \%$ fiber volume, a resin viscosity of $0.1 \mathrm{~Pa} \mathrm{~s}$, and a constant flow rate of $0.5 \mathrm{~cm}^{3} / \mathrm{s}$.

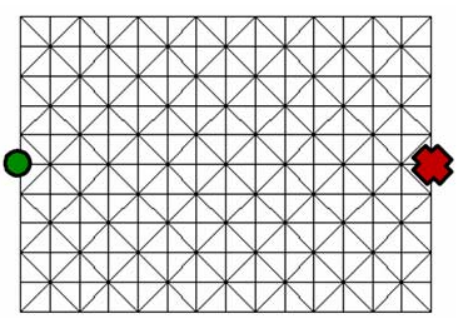

Uniform Permeability Case1a

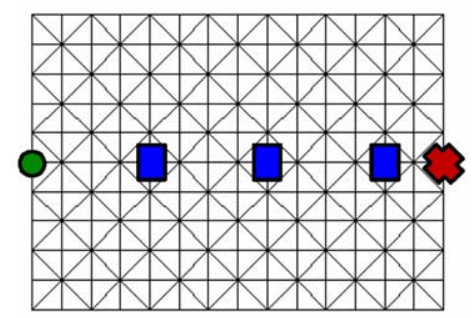

Uniform Permeability

Case1b

Fig. 10. Case 1. Uniform permeability and induced variations in the incubation time at the injection nozzle.

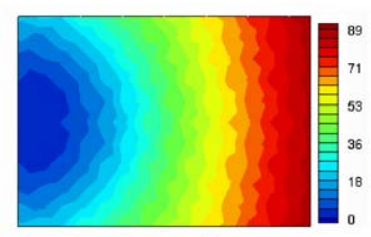

Case1a. Uniform Perm.

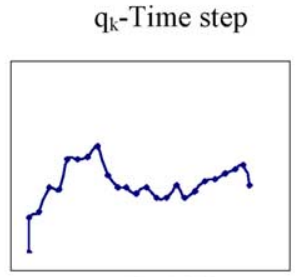

$\mathrm{Q}=1.09$

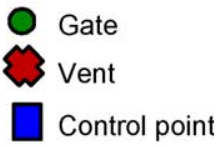

Dispersion index Incubation Time in nodes

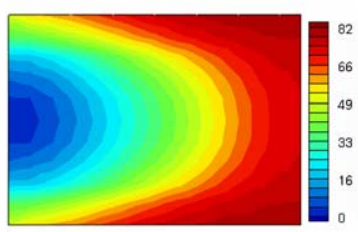

Fig. 11. Simulation results for case 1a.

Flow Pattern

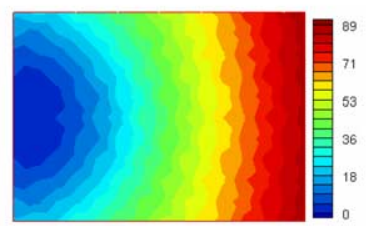

Case1b. Uniform Perm.
$\mathrm{q}_{\mathrm{k}}$-Time step

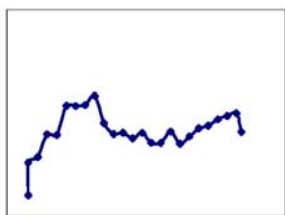

$\mathrm{Q}=1.09$
$D_{i}$ in nodes

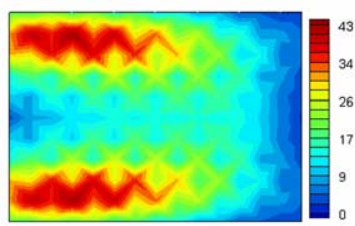

$\mathrm{QD}=18.85$

Fig. 12. Simulation results for case $1 b$. 


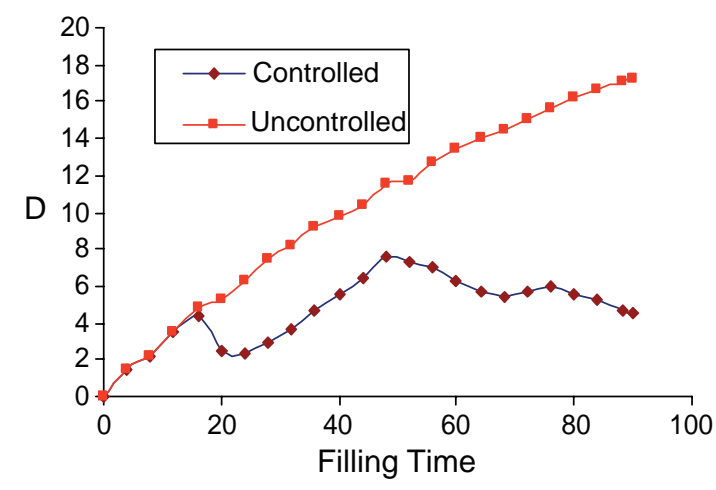

Fig. 13. Time evolution of the resin incubation time dispersion index $D$ for case 1a (uncontrolled) and case $1 \mathrm{~b}$ (controlled).

\subsection{Case with induced variations in the incubation time}

Case 1 refers to a simple injection example with one inlet and one vent in a mold with uniform permeability, see Fig. 10. In case 1a, the resin is injected with constant inhibitor concentration during the filling process with the injection system represented in Fig. 2. We observe in Fig. 11 the flow pattern, the intermediate flow front index $q_{k}$ evolution, the incubation time $E$ distribution and the dispersion index $D_{i}$ profile throughout the mold. We observe that the regions where the resin velocity is smaller (upper and lower regions of the mold) correspond to the highest values for the dispersion index $D_{i}$ due to the differences of the incubation time of the resin located nearby. In that case, the process performance index defined by Eq. (13), takes the value $Q=1.09$ and the new process performance index defined by Eq. (14), $Q D=18.85$.

In the situation depicted in Fig. 10, case 1b, three control points located in the mold are used in the simulations to change the incubation time of the resin located in the injection nozzle as the fluid fills the cavity. One could expect that an appropriate change (according to the chemical kinetics) in the inhibitor concentration could be equivalent to adjust the incubation time of the fluid entering into the mold. Thus, the gate incubation time is set to be the incubation time existing at the control point when the fluid flow front reaches this point. A similar sensor-based control technique was implemented with satisfactory results by Comas-Carmona et al. in [10]. It can be observed in Fig. 12 that the index defined in Eq. (14) $Q D=5.00$ is better in this case as was expected, and $Q$ does not account for this improvement. The uniform color map of the dispersion index indicates an expected homogeneous curing. Fig. 13 shows the evolution of the resin incubation time dispersion index $D$ for both cases. The three peaks in the controlled case correspond to the instants that the incubation time at the injection nozzle is changed.

\subsection{Case of non-homogeneous preform permeability}

Non-uniform preform permeability induces important variations in the flow pattern during the filling process. Three cases with different permeability conditions $(10,50$, 0.1 and 0.02 times the uniform permeability previously used) as shown in Fig. 14 have been analyzed. The associated results are shown in Fig. 15. It can be noticed that without changes in the incubation time (that is equivalent to use the same resin formulation) at the gate during filling, both index $Q$ and $Q D$ agree that case 2a represents the best filling conditions, and case $3 b$ the worst one according with the results obtained for similar conditions in [23].

\subsection{Application in multi-component injection systems}

Case 4 depicted in Fig. 16 analyzes the effect of a high permeability area in the lower part of the mold, which induces a disturbance in the flow pattern and produces a dry spot in the manufactured part due to a wrong location of the vent as depicted in Fig. 17. To avoid that problem other injection gate can be considered as it is depicted in Fig. 18 which opens only when a sensor previously located detects this anomalous behavior. Many research studies have been done showing the application of control strategies in RTM manufacturing $[28,29]$. In this example, the location of this auxiliary gate has been obtained from [30]. The lower race tracking originates a dry spot in the upper right mold corner, which could be corrected with the auxiliary gate. That gate injects resin when a control point located appropriately
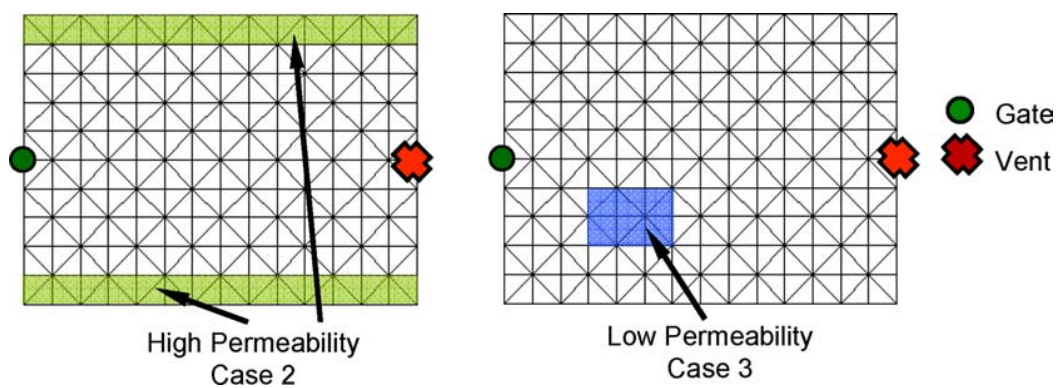

Fig. 14. Case 2 and case 3. Non-homogeneous preform permeability. 


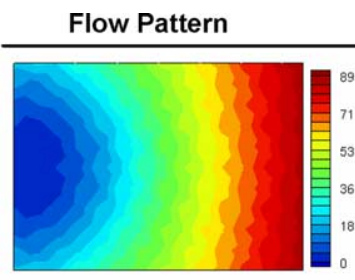

Case1a. Uniform Permeability

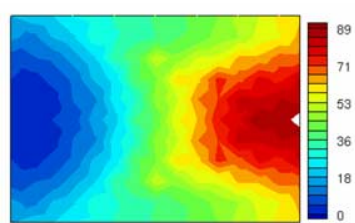

Case2a. Race tracking

(10x)

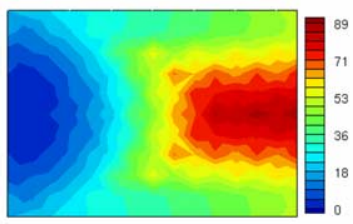

Case2b. Race tracking

(50x)

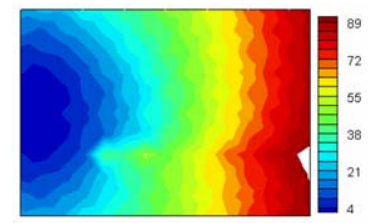

Case3a. Low permeability

$0.1 \mathrm{x}$

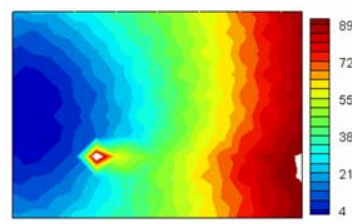

Case3b. Low permeability $0.02 x$

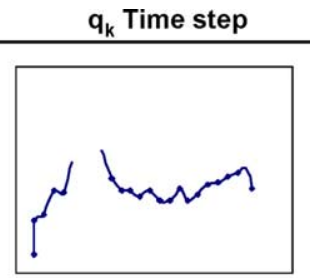

$Q=1.09$

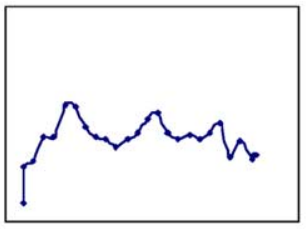

$Q=0.97$

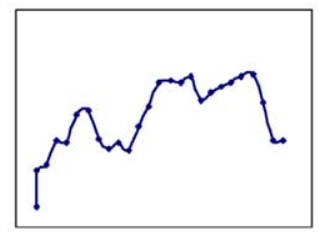

$Q=1.44$

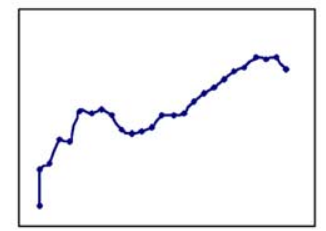

$Q=1.61$

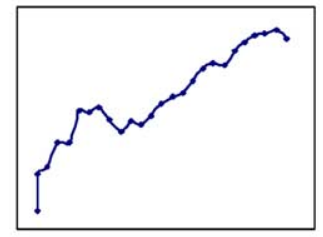

$Q=1.79$
Incubation Time in nodes
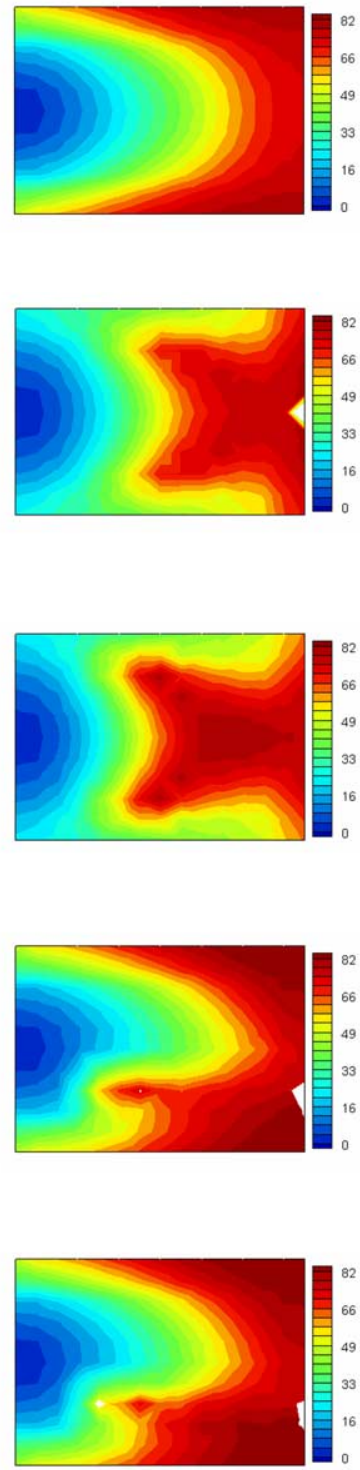

$Q D=33.55$
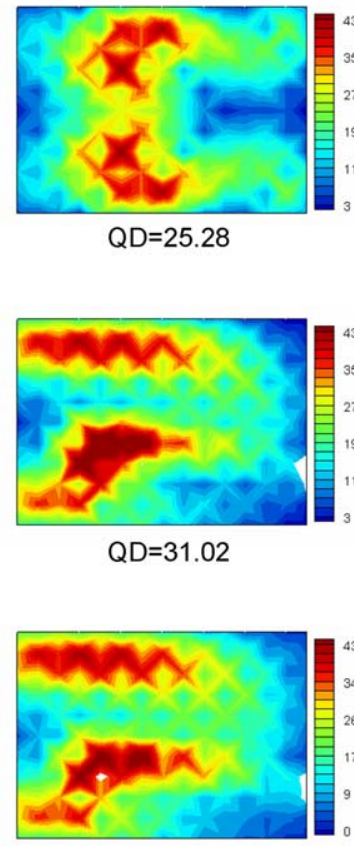

$Q D=25.28$

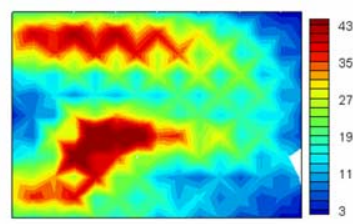

$Q D=31.02$

Fig. 15. Results for different non-homogeneous preforms.

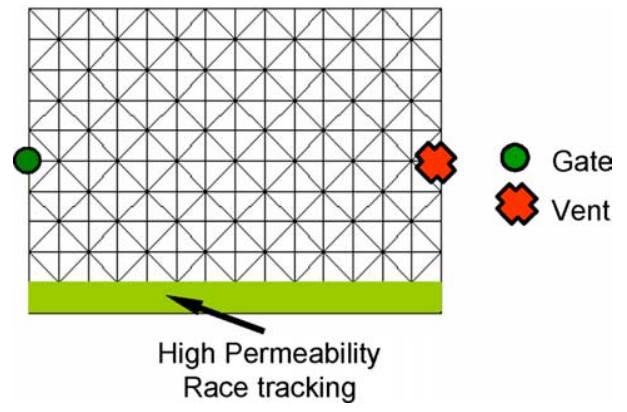

Fig. 16. Case 4. Race tracking in the lower part of the mold.

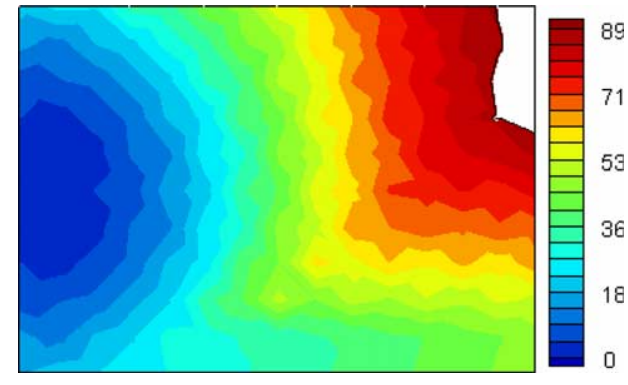

Fig. 17. Flow pattern without auxiliary gate showing the incomplete mold filling. 


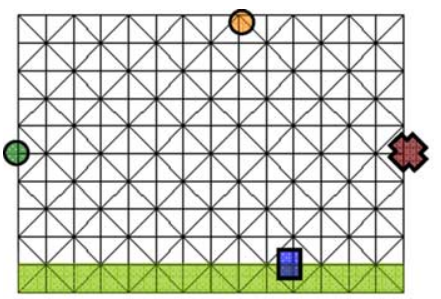

Race tracking

Case 4

Fig. 18. Case 4. Race tracking and auxiliary gate.

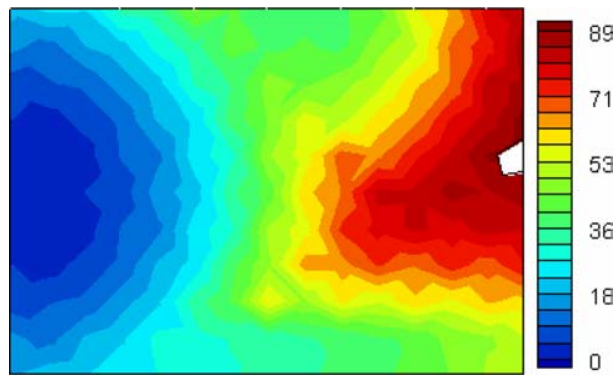

Fig. 19. Flow pattern modified with the inclusion of an auxiliary gate.

detects the unbalanced flow front (see Fig. 19) avoiding the mold-filling defect.

However, in the case depicted in Fig. 19, a problem arises during the welding of the resin flow fronts with different incubation times. Fig. 20 shows that the resin incubation time and the dispersion index $D_{i}$ vary strongly throughout the mold with the use of multiple injection gates where the inhibitor concentration is taken as constant (that is the incubation time is the same on all the gates during the mold filling). We observe areas with a high gradient in the incubation time and in the dispersion index. In that case, the process performance index takes a value of $Q D=36.67$.

Fig. 21 depicts a better solution characterized by $Q D=19.26$, obtained by using different incubation times at the gates during the filling using the same strategy as before. We can notice that the incubation time distribution varies smoothly throughout the mold predicting therefore, lower variations in the curing conversion rates.

\section{Conclusions}

The index proposed in this paper allows the consideration of both resin flow and curing issues in process design optimization. Another advantage of using this index in process optimization is that the computation involved is very efficient as only a two-dimensional calculation is required instead of more complex models requiring the simulation of the coupled flow-thermal models that involve $3 \mathrm{D}$ or $2.5 \mathrm{D}$ computations. This index seems to be very useful in LCM process design optimization where costly iterative stochastic search algorithms such as genetic algorithms are used and could be easily adapted to a specific resin system using its rheokinetic behaviour. This last topic has not been addressed in the present work. Moreover, the comparison between numerical predictions and experiments is a work in progress.
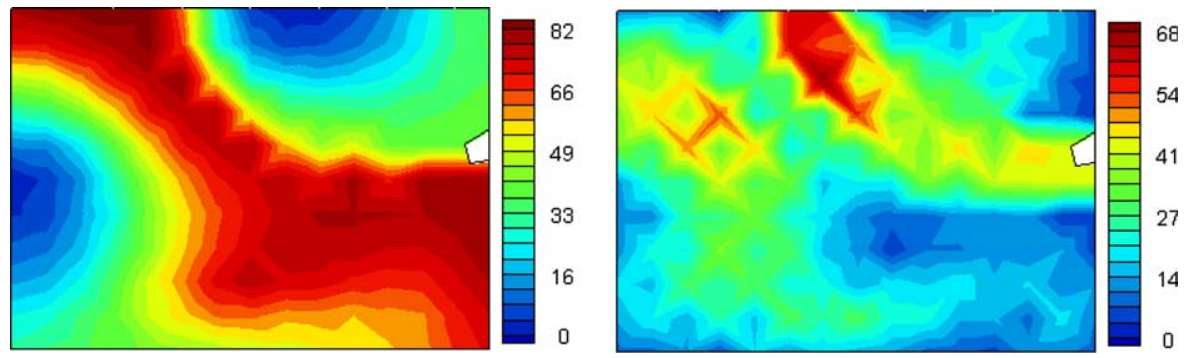

Fig. 20. Incubation time (left) and dispersion index $D_{i}$ in nodes (right) without inhibitor concentration variation at the injection gates.
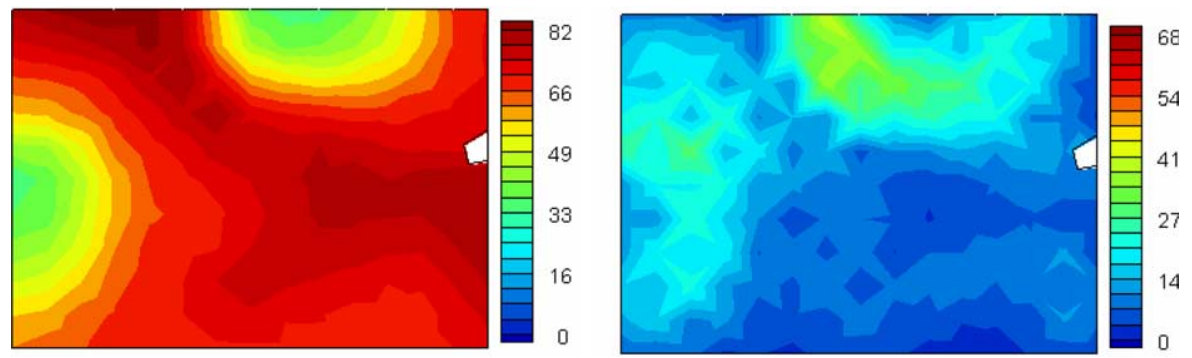

Fig. 21. Incubation time (left) and dispersion index $D_{i}$ in nodes (right) with inhibitor concentration variations at the injection gates. 


\section{Acknowledgements}

This research work is supported by a grant from the spanish Ministerio de Ciencia y Tecnología (MCYT), project DPI2004-03152 and by the project PRUCH-03/08 of the University Cardenal Herrera-CEU.

\section{References}

[1] García JA, Gascón L, Chinesta F. A fixed mesh numerical method for modelling the flow in liquid composites moulding processes using a volume of fluid technique. Comput Methods Appl Mech Eng 2003; 192(7-8):877-93.

[2] Hirsch C. Numerical computation of internal and external flows. Computational methods for inviscid and viscous flows. vol. 2. Chichester: Wiley; 1990.

[3] Leveque R. Numerical methods for conservation laws. Basel: Birkhäuser; 1990.

[4] Harten A. High resolution schemes for hyperbolic conservation laws. J Comput Phys 1983;49:357-93.

[5] Sweby P. High resolution schemes using flux limiters for hyperbolic conservation conservation laws. SIAM J Numer Anal 1984;995-1011.

[6] Sánchez F. Propuesta de un esquema numérico eficiente para el tratamiento de los problemas de transporte en la simulación del moldeo por transferencia de resina. PhD Thesis, Universidad Politécnica de Valencia; 2004.

[7] Ramis X, Salla JM. Efect of the inhibitor on the curing of an unsaturated polyester resin. Polymer 1995;36(18):3511-21.

[8] Rouison D, Sain M, Couturier M. Kinetic study of an unsaturated polyester resin containing an inhibitor. J Appl Polym Sci 2003;89: 2553-61.

[9] Cardona C, Ziaee S, Advani SG. Spatially homogeneus gelation in liquid composite molding. Polym Eng Sci 2002;42(8):1667-73.

[10] Cardona C, Ziaee S, Advani SG. Etude et contrôle de la polimérisation homogène dans les procédés LCM. Revue des Composites et Matériaux Avancés 2003;13.

[11] Dunkers J, Flyn KM, Parnas RS. A mid infrared attenuated total internal reflection cure sensor for control of resin transfer moulding of a pre-ceramic. Compos Part A 1997;28(A):163-70.

[12] Gascón L, Sánchez F, García JA, Chinesta F. A flux limiter technique for the calculation of the incubation time in LCM. In: Proceedings of ESAFORM conference, S. Stören; April 2004. p. 101-4.

[13] Sánchez F, Gascón L, García JA, Chinesta F. Some numerical schemes for the numerical treatment of the advection equation in Liquid Composite Moulding processes. In: Proceedings of IPCM-7 Conference, Delaware; July 2004. p. 263-8.
[14] Chinesta F, Mabrouki T, Ramón A. Some difficulties in the flow treatment in fixed mesh simulations of composites forming processes. In: Proceedings of ESAFORM; 2002.

[15] García JA, Gascón L, Sánchez F, Chinesta F. Mould filling simulation in RTM processes. Revue des Composites et Matériaux Avancés 2003;3(1):85-98.

[16] Bruschke M, Advani SG. A numerical approach to model nonisothermal viscous flow through fibrous media with free surface. Int J Numer Methods Fluids 1994;19:575-603.

[17] Lin R, Lee LJ, Liou M. Non-isothermal mold filling and curing simulation in thin cavities with preplaced fiber mats. Int $\mathbf{J}$ Polym Process 1991;6:357-69.

[18] Trochu F, Gauvin R, Gao DM. Numerical analysis of the resin transfer moulding process by the finite element method. Adv Polym Technol 1993;12(4):329-42.

[19] Young WB. Three dimensional nonisothermal mold filling simulations in RTM. Polym Compos 1994;15(2)

[20] Antonucci V, Giordano M, Nicolais L. A simulation of the nonisothermal resin transfer molding process. Polym Eng Sci 2000;40(12).

[21] Rudd CD, Rice EV, Bulmer IJ, Long AC. Process modelling and design for resin transfer molding. Plast Rub Compos Process Appl 1993.

[22] Jiang S, Zhang C, Wang B. Optimum arrangement of gate and vent locations for RTM process design using a mesh distance-based approach. Compos Part A 2002;33:471-81.

[23] Jiang S, Zhang C, Wang B. A process performance index and its application to optimization of the RTM process. Polym Compos 2001; 22(5):690-701.

[24] Mathur R, Fink BK, Advani SG. Use of genetic algorithms to optimize gate and vent locations for the resin transfer molding process. Polym Compos 1999;20(2):67-78.

[25] Spoerre J, Zhang C, Wang H, Parnas E. Integrated product and process design for resin transfer molded parts. J Compos Mater 1998;28(12): 98-113.

[26] Luo J, Liang Z, Zhang C, Wang B. Optimum tooling design for resin transfer molding with virtual manufacturing and artificial intelligence. Compos Part A 2001;32:877-88.

[27] Gokce A, Hsiao K, Advani SG. Branch and bound search to optimize injection gate locations in liquid composite molding processes. Compos Part A 2002;33:1263-72.

[28] Bickerton S, Stadtfeld HC, Steiner KV, Advani SG. Design and application of actively controlled injection schemes for resin-transfer molding. Compos Sci Technol 2001;61:1625-37.

[29] Lawrence JM, Hsiao K-T, Don RC, Simacek P, Estrada G, Sozer EM, Stadtfeld HC, Advani SG. An approach to couple mold design and online control to manufacture complex composite parts by resin transfer molding. Compos Part A 2002;33:981-90.

[30] Gokce A, Advani SG. Simultaneous gate and vent location optimization in liquid composite molding processes. Compos Part A: Appl Sci Manuf 2004;35:1419-32. 